\title{
Evolución de la lactancia materna en la población que atiende el Centro de Salud de Mendillorri
}

\section{Evolution of breastfeeding in the population attending the Mendillorri Health Centre}

\author{
I. Baztán ${ }^{1}$, I. Ortega ${ }^{1}$, Y. Armendáriz ${ }^{2}$, E. Barace ${ }^{1}$
}

\section{RESUMEN}

Este estudio tiene como objetivo estimar cuál es la prevalencia y duración de la lactancia materna y cómo ha variado en la población que atiende al centro de salud de Mendillorri entre los años 2002 y 2006. Se estudiaron dos cohortes de neonatos (nacidos en 2002 y nacidos en 2006), que acudían a nuestro centro, mediante revisión de la historia clínica informatizada. Se comparan las cifras de prevalencia de las diversas categorías de lactancia en el momento del alta de la maternidad, y con periodicidad mensual, hasta el primer año de vida. Se registra también si se realizó la visita domiciliaria de enfermería.

De los resultados obtenidos se puede concluir que el panorama de la lactancia materna ha cambiado sustancialmente en esta zona tras al alta hospitalaria, demostrándose que las cifras de prevalencia a lo largo del primer año de vida son significativamente superiores en 2006 versus 2002.

Palabras clave. Lactancia Materna. Prevalencia. Atención Primaria. Intervención. Profesionales sanitarios.

\begin{abstract}
The aim of this study is to estimate the prevalence and duration of breastfeeding, and how this has varied in the population attending the Mendillorri health centre between the years 2002 and 2006 . We studied two cohorts of neonates (born in 2002 and 2006, respectively) who attended our centre, through a revision of their computerised clinical history. The prevalence figures of the different lactation categories were compared, with a monthly periodicity from the time of maternity discharge until the first year of life. Home visits by nurses were also registered where these occurred.

The conclusion drawn from the results is that the panorama of maternal lactation has changed substantially in this area following hospital discharge; the results show that the prevalence figures over the first year of life are significantly higher in 2006 compared with 2002 .
\end{abstract}

Key words. Brestfeeding. Prevalence. Primary care. Intervention. Health professionals.
1. Centro de Salud de Mendillorri. Servicio Navarro de Salud-Osasunbidea.

2. Hospital Miguel Servet. Zaragoza.

Recepción el 18 de septiembre de 2008 Aceptación provisional el 10 de octubre de 2008 Aceptación definitiva el 11 de noviembre de 2008

\section{Correspondencia:}

Iris Baztán Arrastia

Paseo Ibaialde, 166

31192 Mutilva Baja (Navarra)

Tfno. 948072053

E-mail: Irisbaztan@hotmail.com 


\section{INTRODUCCIÓN}

La promoción de la lactancia materna (LM) es una de las estrategias prioritarias para el fomento y la protección de la salud en todo el mundo. Así lo han reconocido la Organización Mundial de la Salud (OMS), UNICEF $^{1,2}$ y diversas organizaciones científicas $^{3-5}$. Las ventajas de la LM van desde los aspectos nutricionales e inmunitarios hasta los preventivos y psicológicos ${ }^{5-8}$. Sin embargo, pese a las recomendaciones de la OMS de mantener como patrón de oro de la alimentación infantil la lactancia materna exclusiva (LME) hasta los seis meses y posteriormente, junto con otros alimentos, hasta los dos años de vida, en nuestro medio estas expectativas no se cumplen.

En España, tal y como se pone de manifiesto en los estudios de los últimos años, las cifras de lactancia natural (LN) en el momento del alta de la maternidad se mantienen constantes desde la década de 1990 , en torno al $80-90 \%^{8-10}$. Sin embargo, a lo largo del primer semestre de vida es cuando la LM se abandona masivamente, de tal modo que a los seis meses, siendo muy variable la cifra según los diversos estudios, se sitúa en la mayoría de éstos por debajo del $30 \%$.

En general, las madres españolas y también las madres navarras tienen una idea positiva respecto de la lactancia materna, ya que como hemos resaltado, tan sólo el $10 \%$ decide no amamantar a sus hijos. Sin duda, el mayoritario inicio de la lactancia materna y el prematuro abandono de ésta se debe a factores relacionados con el entorno que rodea a la madre y que no favorecen suficientemente su continuidad.

En Navarra, al igual que en el resto de comunidades autónomas de nuestro país, se han incluido estrategias para la promoción de la lactancia materna. Sin embargo, éstas no han sido suficientes y se ha demostrado que es imprescindible que las personas que participen en el cuidado de la madre y de su hijo sean capaces de transmitir la conveniencia de la lactancia natural, facilitar su inicio y su mantenimiento, ayudando a soslayar las frecuentes dificultades que surgen, asesorándoles de forma profesional ${ }^{11-16}$.

En el presente trabajo se estudia la prevalencia y duración de la LM en el centro de salud de Mendillorri y cómo ha variado entre los años 2002 y 2006.

\section{MATERIAL Y MÉTODOS}

El centro de salud de Mendillorri atiende a un total de 13.842 habitantes (datos de abril 2006), que viven mayoritariamente en dos núcleos poblacionales vecinos diferenciados: Mendillorri (barrio periférico de Pamplona) y Sarriguren (población de reciente creación perteneciente al Valle de Egüés). Se trata de una población de carácter urbano, de nivel socioeconómico medio-bajo, eminentemente joven, con un 37\% de población menor de 14 años, y con un índice de nacimientos anuales que supera los 200. Hay que destacar que el porcentaje de inmigrantes es bajo (un 4,8\% sobre el total), comparado con el del resto de Navarra (9,2\% en 2007).

Se diseñó un estudio descriptivo retrospectivo, cuya población base fueron todos los niños nacidos en la zona en el año 2002 $(n=235)$ y en el año $2006(n=237)$. La fuente de datos se ha obtenido de la base de datos de usuarios adscritos al centro de salud.

Los criterios de inclusión han sido niños nacidos en el primer semestre del año $2002(n=123)$ y del mismo semestre del año $2006(n=112)$, que acudían a nuestro centro de salud en el primer año de vida. Se han elegido estos semestres con el fin de que los niños nacidos en el 2006 hubieran cumplido un año en el momento de la revisión de las historias clínicas.

Los criterios de exclusión fueron: síndrome de Down, gemelos, nacidos con menos de $2.500 \mathrm{~g}$, ingreso neonatal por cualquier motivo y niños que en las primeras visitas acudieron a otro centro de salud.

Se consideraron válidos 94 recién nacidos del primer semestre del $2002(76,42 \%)$ y 86 del mismo semestre del año 2006 (76,78\%). Tanto los casos incluidos como los excluidos se muestran en la tabla 1 .

La recogida de datos se ha realizado mediante la revisión de la historia clínica informatizada (este método anula el sesgo de recuerdo en el que incurre el método de encuesta telefónica). Se diseñó una plantilla para recuperar la información relativa al tipo de alimentación que recibía el recién nacido ( $\mathrm{RN}$ ) en la visita domiciliaria (si la 
hubo o no entre los 5 y 10 primeros días de vida) y en los exámenes de salud de los 15 días, 1 mes, 2, 3, 4, 5, 6, 9 y 12 meses. En los casos en los que no había registro en las últimas visitas se les llamó por teléfono (sólo en un caso). Las variables estudiadas fueron dos:

1. Tipo de lactancia (variable principal) con tres categorías: lactancia materna exclusiva (LME), lactancia mixta (LMIXTA) y lactancia artificial exclusiva (LAE) al alta hospitalaria, un mes, $2,3,4,5,6,9$ y doce meses.
2. La existencia o no de visita domiciliaria de Enfermería de Pediatría en consulta en el centro de salud y/o en domicilio tras el alta hospitalaria y entre los 5 y 10 primeros días de vida del recién nacido $(\mathrm{RN})$.

El análisis de los datos se llevó a cabo comparando la prevalencia de la lactancia en cada mes de estudio entre los dos años utilizando la prueba del Chi cuadrado, la prueba exacta de Fisher y la t de Student de comparación de medias. Se ha establecido el nivel de significación en el $5 \%(0,05)$.

Tabla 1. Número de casos incluidos y excluidos.

\begin{tabular}{|c|c|c|c|c|c|c|c|c|}
\hline & $\begin{array}{l}\text { Síndrome de } \\
\text { Down }\end{array}$ & Gemelos & $\begin{array}{c}\text { Menores de } \\
2.500 \mathrm{~g}\end{array}$ & $\begin{array}{c}\text { Ingreso } \\
\text { neonatal por } \\
\text { cualquier } \\
\text { motivo }\end{array}$ & $\begin{array}{c}\text { Niños que no } \\
\text { acudieron a } \\
\text { las primeras } \\
\text { visitas en } \\
\text { nuestro } \\
\text { centro }\end{array}$ & $\begin{array}{c}\text { Total } \\
\text { excluidos }\end{array}$ & $\begin{array}{c}\text { Total } \\
\text { incluidos }\end{array}$ & Total \\
\hline $\begin{array}{c}\text { Nacidos en } \\
\text { el primer } \\
\text { semestre } \\
2002\end{array}$ & 0 & 4 & 6 & 0 & 19 & 29 & $\begin{array}{c}94 \\
(76,42 \%)\end{array}$ & 123 \\
\hline $\begin{array}{c}\text { Nacidos en } \\
\text { el primer } \\
\text { semestre } \\
2006\end{array}$ & 1 & 8 & 0 & 2 & 15 & 26 & $\begin{array}{c}86 \\
(76,78 \%)\end{array}$ & 112 \\
\hline
\end{tabular}

\section{RESULTADOS}

La media de duración de la LM fue de 105 días en 2002 y 180 días en 2006 y para la LME de 86 días en 2002 y 101 días en 2006. Estas diferencias fueron significativas para ambos tipos de lactancias $(\mathrm{p}<0,05)$.

En el momento del alta de la maternidad, comparando la diferencia de las distintas lactancias (Figs. 1 y 2), se observa cómo la incidencia de LME es superior en el año 2002, 85\% frente al 81\% de 2006. La LAE es similar en los dos años (11\% y $10 \%$ respectivamente). A partir del mes de vida, se observa que tanto las cifras de LME como las de lactancia materna (LM: LME+LMIXTA) son superiores siempre en el año 2006.

La diferencia entre los dos años estudiados aumenta progresivamente (siempre a favor de 2006) a medida que se avanza en la edad del niño, desde el 13\% para la LME y $8 \%$ para la LM en el primer mes hasta el $23 \%$ para la LME y $31 \%$ para la LM en el sexto mes (Figs. 1 y 2). Se puede hablar de diferencias realmente significativas a partir del cuarto mes para la LME y del sexto mes para la LM. Es decir, hay un cambio significativo en el comportamiento de las madres, se produce un descenso significativo de la lactancia artificial a partir del $4^{\circ}$ mes entre los dos períodos de tiempo.

En 2006, tanto la LME como la LM se mantienen más o menos constantes a lo largo de los tres primeros meses $(72-81 \%$ y $85-90 \%$ respectivamente), descendiendo hasta un $36 \%$ para la LME y $64 \%$ para la LM en el sexto mes de vida.

Se constata así mismo la tendencia a prolongar la LM a lo largo del primer año de vida de las madres de esta zona, dado que a los nueve meses de vida continúan 
con LM el $45 \%$ de los niños, frente al 18\% de 2002. A su vez, al año de vida, en 2006 per- manecen con LM el 28\% de los niños frente al $10 \%$ de 2002 (Figs. 1 y 2).

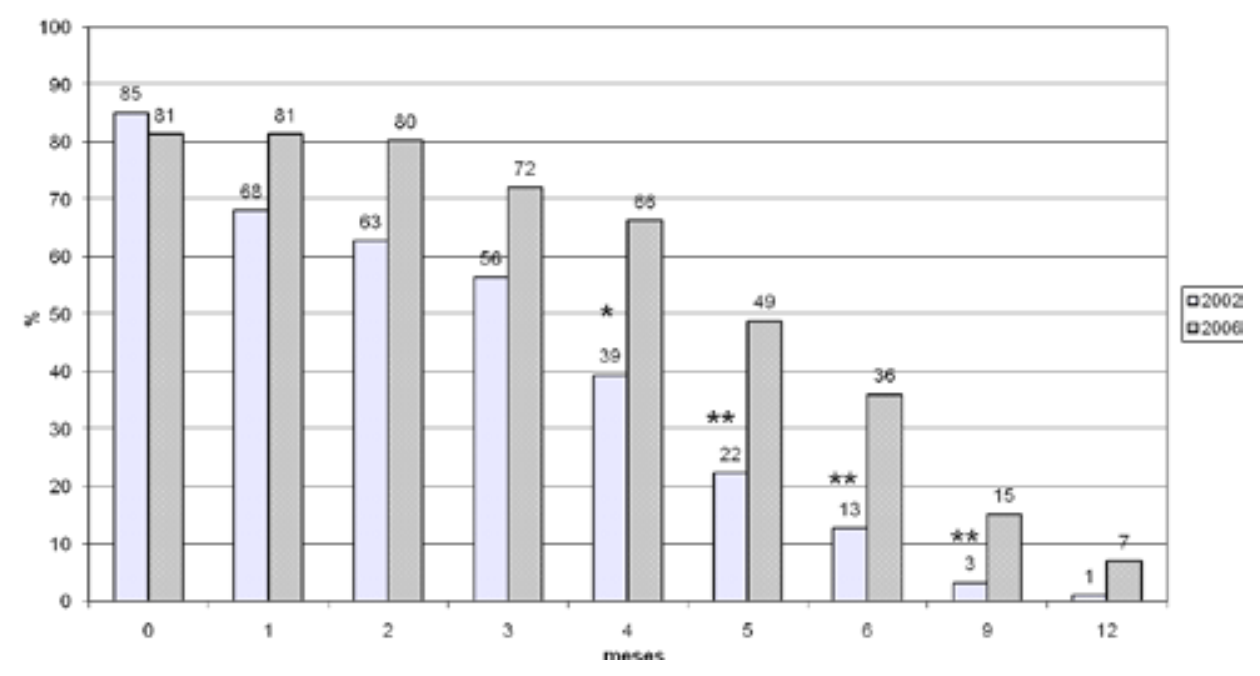

Figura 1. Evolución de la prevalencia de la lactancia materna exclusiva 2002-2006.

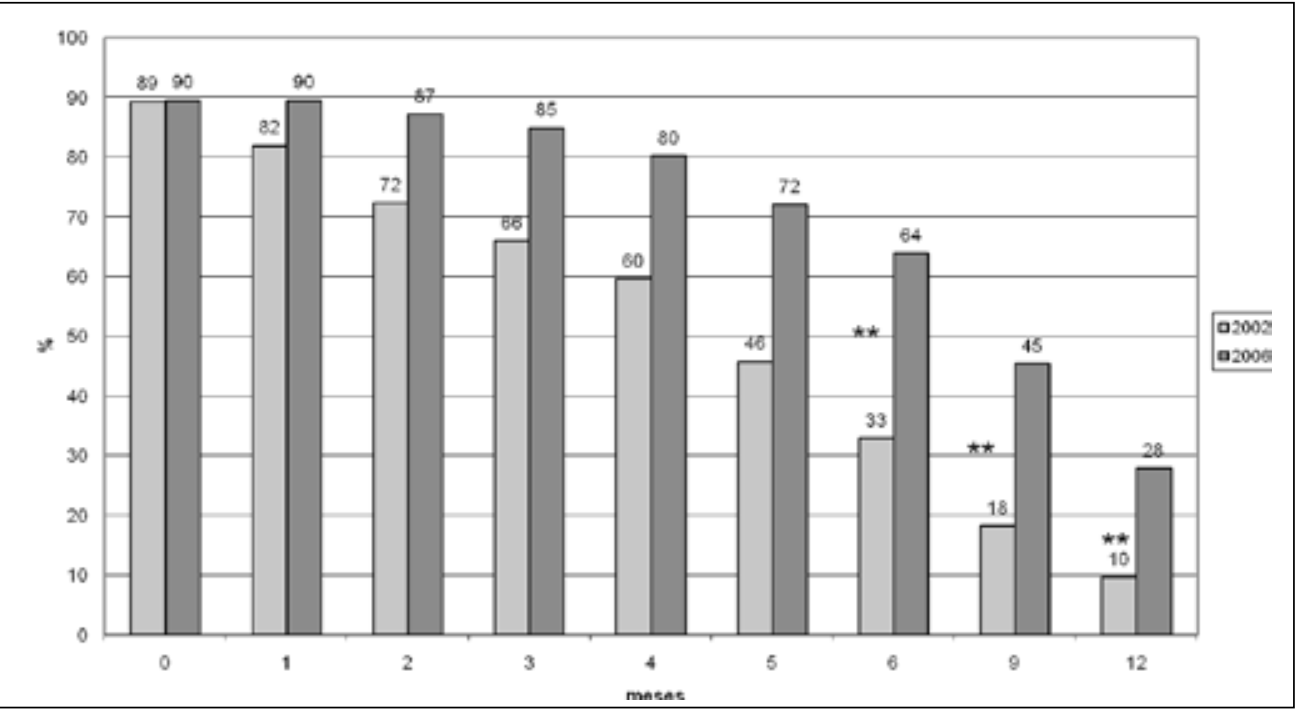

Figura 2. Evolución de la prevalencia de la lactancia materna: LME + L. Mixta 2002-2006 


\section{DISCUSIÓN}

En nuestra población el $90 \%$ de madres decide iniciar LM, dato que se mantiene más o menos constante en Navarra ${ }^{10}$ desde la década de los años 1990. En el mantenimiento de esta cifra influye de forma decisiva la decisión materna de alimentar a su hijo con leche materna, decisión que se debe, en muchos casos, al aumento en general del conocimiento de los beneficios de la LN que tienen las madres y padres, en parte gracias a los programas de "preparación al parto" impartidos por matronas $^{7,17-19}$.

Por el contrario, comparando las cifras de 1993 en Navarra ${ }^{10}$ con las de nuestro trabajo, podemos comprobar que el panorama de la LN ha cambiado sustancialmente en nuestra zona tras el alta hospitalaria ya que a los seis meses la prevalencia ha aumentado significativamente del $11 \%$ al $64 \%$ en estos trece años. Por tanto, se constata un esperanzador resurgir de las cifras de LM ya en 2002 pero sobre todo en 2006 en el primer semestre de vida. Estos datos nos sitúan en 2006 en un nivel más alto respecto de la mayoría de los estudios realizados en nuestro país ${ }^{9,10,17,18,20-22}$ y en niveles ligeramente por debajo (similares en los cuatro primeros meses y ligeramente inferiores en los meses sucesivos) al del estudio del impacto de la IHAN $^{23}$ de Murcia. Esta tendencia ascendente de prolongar la LM se objetiva también en el comportamiento de la prevalencia en el segundo semestre de vida. Estos resultados, siendo optimistas, distan todavía de alcanzar las recomendaciones de la OMS.

El considerable aumento de la duración de la lactancia materna entre los años 2002-2006 en nuestra zona (la diferencia es del $23 \%$ para la LME y del $31 \%$ para la LM a los seis meses), puede ser en parte atribuible a la intervención que hacen las enfermeras de pediatría de nuestro centro de salud. En nuestro centro en el año 2002 y tras el alta hospitalaria, las madres no recibían el apoyo de la enfermera de pediatría en los primeros días de vida del recién nacido. Este servicio se ofrece desde el año 2004: consiste en un primer contacto telefónico con la madre por parte de los profesionales de enfermería en el momento en que éstos reciben la notificación del nacimiento -suele ser alrededor del quinto o sexto día de vida-. En la misma conversación, además de un primer tanteo sobre las dificultades y problemas en el inicio de la lactancia, se concierta cita lo más próxima posible en consulta o domicilio para la valoración del RN y resolución de problemas. Tras este primer contacto con el servicio de enfermería de pediatría del centro de salud, se oferta apoyo telefónico y/o en consulta a demanda en cualquier momento según las necesidades y dificultades de cada madre.

Numerosos estudios reflejan que la intervención de los profesionales sanitarios $^{11-16,23-25}$ y en particular el consejo profesional de la enfermera pediátrica ${ }^{18,26,27}$ es un factor predictor positivo para el éxito de la LM.

Una vez iniciada la LM, la continuidad de ésta depende muchas veces de las dificultades que surgen en los primeros días del puerperio: irritabilidad del bebé, dolor por grietas, depresión post-parto, estrés ante la nueva responsabilidad, ideas negativas del entorno más cercano respecto de la LN, la propia inexperiencia de la madre (no debe olvidarse, que la técnica de amamantamiento, transmitida de madres a hijas a lo largo de la historia, se ha perdido en las últimas generaciones).

En el abordaje de muchas de estas dificultades, la enfermera de pediatría del centro de salud se encuentra en una situación privilegiada para influir de forma positiva con su actuación. Es por ello, que estos profesionales, deben ser conscientes de la necesidad de mantener actualizada la formación sobre LM y participar activamente en el apoyo y promoción de ésta, dada la importancia que su intervención tiene, en los primeros días tras el alta de la maternidad, en la alimentación del niño de forma natural.

En esta línea, se aconseja realizar un control precoz por parte de la enfermera de pediatría del centro de salud (a las 48 horas tras el alta de la maternidad), tal y como lo propone Pallas en el "Manual de actividades preventivas en la infancia y adolescencia" . En Navarra, las enfermeras contamos con una metodología de trabajo propia, donde están muy desarrollados los cuidados al RN y en especial el apoyo a la $\mathrm{LN}$, a través de la visita domiciliaria ${ }^{28}$, pero quizás sería conveniente llevarla a cabo alrededor del quinto día, en lugar del décimo 
(como se viene realizando actualmente), con el fin de comprobar la instauración correcta de la LM y resolver los problemas, dificultades y signos inadecuados de amamantamiento que puedan llevar al abandono precoz de la LN.

Al mismo tiempo sugerimos, que sería recomendable ofrecer desde los servicios de pediatría de los centros de salud, para todos aquellos padres que lo deseen, una visita prenatal, cercana al nacimiento, con la enfermera pediátrica, con el objetivo principal de informar sobre los beneficios de la LN y de recordar la técnica y claves para su correcta instauración. De este modo se refuerza lo aprendido en el curso de preparación al parto, al tiempo que se establece un primer contacto con el equipo de pediatría encargado del cuidado del futuro RN.

En conclusión, se objetiva un significativo descenso de la LA a partir del $4^{\circ}$ mes de vida y el mantenimiento casi uniforme (en torno al $78-90 \%$ ) de la prevalencia de lactancia natural en los primeros 4 meses de vida, momento en el cual se constatan que se inician muchas de las lactancias mixtas. Este hecho coincide con el inicio de la reincorporación laboral materna, por lo que se hace imprescindible incidir en la información y apoyos para que las madres puedan compatibilizar y simultanear la vuelta al trabajo con la lactancia natural.

\section{Agradecimientos}

A Idoia Gaminde Inda del Servicio de Docencia, Investigación y Desarrollo Sanitarios del Departamento de Salud por su colaboración en la elaboración de este trabajo.

\section{BIBLIOGRAFÍA}

1. OMS-UNICEF, Declaración Innocenti. WHO. Florencia 1990, que fue adoptada por la $45^{\mathrm{a}}$ Asamblea Mundial de la Salud en mayo de 1992 (resolución WHA 45.34).

2. OMS. Nutrición del lactante y del niño pequeño. Estrategia mundial para la alimentación del lactante y del niño pequeño. Informe de la Secretaría. Géneve: $55^{\mathrm{a}}$ Asamblea Mundial de la Salud. 16 de Abril del 2002. A55/15. Disponible en:

http://ftp.who.int/gb/ebwha/pdf_files/ WHA55/ea5515.pdf.
3. European Comission, Directorate Public Health and Risk Assessment. EU Proyect on Promotion of Breastfeeding in Europe. Protection, Promotion and Support of Breastfeeding in Europe: A blueprint for Action. Luxembourg 2004. Disponible en:

http//europa.eu.int/comm/healt/ph_projects/2002/promotion/promotion_2002_18_ en.htm.

4. Gartner LM, Morton J, Lawrence RA, Naylor AJ, O'Hare D, Schanler RJ et al. American Academy of Pediatrics Section on Breastfeeding Breastfeeding and the Use of Human Milk. Pediatrics 2005; 115: 496-506.

5. Pallás Alonso C. Promoción de la lactancia materna. Alimentación en el primer año de vida. En: Manual de actividades preventivas en la infancia y adolescencia. Noviembre, 2004: 101-127.

6. Barriuso L, De Miguel M, Sánchez M. Lactancia materna: factor de salud. Recuerdo histórico. An Sist Sanit Navar 2007; 30: 383-391.

7. Hernández Aguilar MT. Epidemiología de la lactancia materna. Prevalencia y tendencia de la lactancia materna en el mundo y en España. En: Lactancia materna: Guía para profesionales. Monografías de la AEP 2004; 5: $31-42$.

8. Hernández Aguilar mT, Aguayo Maldonado J. La Lactancia Materna. Cómo promover y apoyar la lactancia materna en la práctica pediátrica. Recomendaciones del Comité de Lactancia de la AEP. An Pediatr 2005; 63 : 340-356.

9. Barriuso LM, Sánchez-Valverde F. Prevalencia de la lactancia materna en el norte de España. An Sist Sanit Navar 1998; 21 (Supl 3): 13-19.

10. BarRiuso LM, SÁnchez-Valverde F. Lactancia materna en Navarra. An Sist Sanit Navar 2002; 25 (Supl 2): 17-23.

11. Dyson L, McCormick F, Renfrew MJ. Intervenciones para promover el inicio de la lactancia materna (Revisión Cochrane traducida). En: La Biblioteca Cochrane Plus, número 2, 2007. Oxford: Update Software Ltd. Disponible en: http://www.update-software.com.

12. Bonuck KA, Trombley M, Freeman K, Mckee D. Randomized, controlled trial of a prenatal and postnatal lactation consultant intervention on duration and intensity of breastfeeding up to 12 months. Pediatrics 2005; 116 : 1413-1426.

13. Kramer MS, Chalmers B, Hodnett ED, Devkovskaya $Z$, Dzikovich I, Shapiro $S$ et al. Breastfeeding intervention trial (PROBIT). A randomized trial in the republic of Belarus. JAMA 2001; 285: 413-420. 
14. Jovani Roda L, Gutiérrez Culsant P, Aguilar Martín C, Navarro Caballé R, Mayor Pegueroles I, JoRnET TORRENT Y. Influencia del personal sanitario de asistencia primaria en la prevalencia de la lactancia materna. An Pediatr 2002; 57: 534-539.

15. Parellada Esquius N, García Casanova MC, PI Juan M, García Casanova S, Ruiz Mariscal E. Lactancia materna: ¿puede el personal sanitario influir positivamente en su duración? Aten Primaria 2005; 35: 295-300.

16. Britton C, McCormick FM, Renfrew MJ, Wade A, KING SE. Apoyo para la lactancia materna (Revisión Cochrane traducida). En: La Biblioteca Cochrane Plus, 2007 Número 2. Oxford: Update Software Ltd. Disponible en: http:// www.update-software.com.

17. Suárez Gil P, Alonso Lorenzo JC, López Díaz AJ, Martín Rodríguez D, Martínez SuÁrez MM. Prevalencia y duración de la lactancia materna en Asturias. Gacet Sanit 2001; 15: 104-110.

18. Morales GIL IM. Empleo de la metodología enfermera en la promoción de la lactancia materna. Universidad de Málaga. IV Congreso español de lactancia materna. Tenerife 2006. Libro de Actas: 5-7

19. Estévez González MD, Martell Cebrián D, Medina Santana R, García Villanueva E, SaAvedra Santana P. Factores relacionados con el abandono de la lactancia materna. An Esp Pediatr 2002; 56: 144-150.

20. Hostaltot Abás AM, Sorní Hubrecht A, Jovaní Roda L, Rosal Roig J, Mercé Gratacós J, Iglesias Niubó J et al. Lactancia materna en el sur de Cataluña. Estudio de los factores socioculturales y sanitarios que influyen en su elección y mantenimiento. An Esp Pediatr 2001; 54: 297-302.
21. Fidalgo Alvárez I, López Palacios D, Jiménez RoDríguez M, GonzÁlez Martínez A, Costales ÁlvaREz A, Blanco Franco MP et al. Factores relacionados con la lactancia materna. Revista Pediatría de Aten Primaria 2002; 16: 27-38.

22. Ortiz Andrelluchi A, Estévez González MD, Díaz Gómez M, Domenech Martínez E, Armas SÁnchez MR, Antúnez N et al. Prevalencia de la lactancia materna en Canarias. IV Congreso español de lactancia materna. Tenerife 2006. Libro de Actas: 74-75.

23. Hernández Martínez M, García de León González R, Oliver Roig A, Mercader Rodríguez B, Peñas Valiente A, Gómez Ortigosa MA. Evaluación del impacto de la IHAN en las tasas de lactancia materna de nuestra área sanitaria. Hospital Virgen del Castillo, Yecla (Murcia). IV Congreso español de lactancia materna. Tenerife 2006. Libro de Actas: 73-74.

24. Kuan LW, Brito M, Decolongon J, Schoettker PJ, Atherton HD, Kotagal UR. Health system factors contributing to breastfeeding success. Pediatrics 1999; 48: 28.

25. Deshrande AD, Gazmararian JA. Breastfeeding education and support: association with decision to breastfeed. Eff Clin Pract 2000; 3: 116-122.

26. Lu MC, Lange L, Slusser W, Hamilton J, Halfon N. Provider encouragement of breastfeeding: evidence from a national survey. Obstet Gynecol 2001; 97: 290-295.

27. Porteus R, Kaufman K, Rush J. The effect of individualized professional support on duration of breastfeeding: a randomized controlled trial. J Hun Lact 2000; 16: 303-308.

28. Servicio Navarro de Salud (SNS-O). Dirección de Atención Primaria. Programa del niño sano. Pamplona 2002. 
